\title{
Correction: Thyroid cancer causing obstruction of the great veins in
} the neck

\author{
Steve L Hyer*1,3, Prasad Dandekar ${ }^{1}$, Kate Newbold ${ }^{1}$, Masud Haq ${ }^{1}$, \\ Kshama Wechalakar ${ }^{1}$, Khin Thway ${ }^{2}$ and Clive Harmer ${ }^{1}$
}

Address: ${ }^{1 T h y r o i d ~ U n i t, ~ R o y a l ~ M a r s d e n ~ H o s p i t a l, ~ F u l h a m ~ R o a d, ~ L o n d o n, ~ S W 36 J J, ~ U K, ~}{ }^{2}$ Department of Pathology, Royal Marsden Hospital, Fulham Road, London, SW3 6JJ, UK and ${ }^{3}$ St Helier Hospital, Wrythe Lane, Carshalton, Surrey, SM5 1AA, UK

Email: Steve L Hyer* - steve.hyer@epsom-sthelier.nhs.uk; Prasad Dandekar - Prasad.Dandekar@rmh.nhs.uk;

Kate Newbold - Kate.Newbold@rmh.nhs.uk; Masud Haq - masudhaq@hotmail.com; Kshama Wechalakar - kshama.wechalekar@gmail.com; Khin Thway - Khin.thway@rmh.nhs.uk; Clive Harmer - cliveharmer@fsmail.net

* Corresponding author

Published: 18 April 2008

World Journal of Surgical Oncology 2008, 6:40 doi:10.1 186/1477-7819-6-40

This article is available from: http://www.wjso.com/content/6/l/40

(C) 2008 Hyer et al; licensee BioMed Central Ltd.

This is an Open Access article distributed under the terms of the Creative Commons Attribution License (http://creativecommons.org/licenses/by/2.0), which permits unrestricted use, distribution, and reproduction in any medium, provided the original work is properly cited.

After publication of this work [1], we noted that we inadvertently failed to include the complete list of all coauthors. The full list of authors has now been added and the Authors' contributions and Competing interests section modified accordingly.

\section{Competing interests}

The authors declare that they have no competing interests.

\section{Authors' contributions}

SLH: Final draft and literature review; PD: Clinical information, initial draft, $\mathrm{KN}$ : Discussion and editing, $\mathrm{MH}$ Clinical information, CT images and interpretation, KT: Pathological images and reports, KW: Scintigram images and interpretation, $\mathrm{CH}$ : Original concept, final editing

All authors read and approved final manuscript

\section{References}

I. Hyer SL, Dandekar P, Newbold K, Haq M, Wechalakar K, Harmer C: Thyroid cancer causing obstruction of the great veins in the neck. World Journal of Surgical Oncology 2008, 6:36. 